\title{
Latent Space Exploration Using Generative Kernel PCA
}

\author{
D. Winant ${ }^{1}$, J. Schreurs ${ }^{1}$, and J.A.K. Suykens ${ }^{1}$ \\ KU Leuven, Department of Electrical Engineering (ESAT), \\ STADIUS Center for Dynamical Systems, Signal Processing and Data Analytics, \\ Kasteelpark Arenberg 10, B-3001 Leuven, Belgium \\ \{david.winant, joachim.schreurs, johan.suykens\}@kuleuven. be
}

\begin{abstract}
Kernel PCA is a powerful feature extractor which recently has seen a reformulation in the context of Restricted Kernel Machines (RKMs). These RKMs allow for a representation of kernel PCA in terms of hidden and visible units similar to Restricted Boltzmann Machines. This connection has led to insights on how to use kernel PCA in a generative procedure, called generative kernel PCA. In this paper, we investigate the use of generative kernel PCA for exploring latent spaces of datasets. New points can be generated by gradually moving in the latent space, which allows for an interpretation of the components. Firstly, we show illustrative examples of this feature space exploration on two datasets. Afterwards, we demonstrate the use of the tool in combination with novelty detection, where the latent space around novel patterns in the data is explored. This helps in the interpretation of why certain points are considered as novel.
\end{abstract}

Keywords: Kernel PCA · Restricted Kernel Machines - Latent Space Exploration.

\section{Introduction}

Latent spaces provide a representation of data by embedding the data into an underlying vector space. Exploring these spaces allows for deeper insights in the structure of the data distribution, as well as understanding relationships between data points. Latent spaces are used for various purposes like latent space cartography [10], object shape generation [16] or style-based generation [7]. In this paper, the focus will be on how the synthesis of new data with generative methods can help with understanding the latent features extracted from a dataset. In recent years, generative methods have become a hot research topic within the field of machine learning. Two of the most well-known examples include variational autoencoders (VAEs) [8] and Generative Adversarial Networks (GANs) 2]. An example of a real-world application of latent spaces using VAEs is shown in [15, where deep convolututional VAEs are used to extract a biologically meaningful latent space from a cancer transcriptomes dataset. This 
latent space is used to explore hypothetical gene expression profiles of tumors and their reaction to possible treatments. Latent space exploration is also used for interpreting GANs, where interpolation between different images allows for the interpretation of the different features captured by the latent space, such as windows and curtains when working with datasets of bedroom images [11. More recently, the concept of latent space exploration with GANs has been further developed by introducing new couplings of the latent space to the architecture of the generative network, this allows for control of local features for image synthesis at different scales in a style-based design [7. These adaptations of GANs are known as Style-GANs. When applied to a facial dataset, the features can range from general face shape and hair style up to eyes, hair colour and mouth shape.

In this paper, kernel PCA is used as a generative mechanism [13. Kernel PCA, as first described in [12, is a well-known feature extractor method often used for denoising and dimensionality reduction of datasets. Through the use of a kernel function it is a nonlinear extension to regular PCA by introducing an implicit, high dimensional latent feature space wherein the principal components are extracted. In 14, kernel PCA was cast within the framework of Restricted Kernel Machines (RKMs) which allows for an interpretation in terms of hidden and visible units similar to a type of generative neural network known as Restricted Boltzmann Machines (RBMs) 3]. This connection between kernel PCA and RBMs was later used to explore a generative mechanism for the kernel PCA [13].

The goal of this paper is to explore the latent feature space extracted by kernel PCA using a generative mechanism, in an effort to interpret the components. This has led to the development of a Matlab tool which can be used to visualise the latent space of the kernel PCA method along its principal components. The use of the tool is demonstrated on two different datasets. As an illustration, feature space exploration is used in the context of novelty detection [5, where the latent space around novel patterns in the data is explored. This to help the interpretation of why certain points are considered as novel.

In Section 2, we will give a brief review on generative kernel PCA. In Section 3 , we describe the tool we developed to perform latent feature space exploration using generative kernel PCA. Subsequently we will illustrate how latent feature space exploration can help in interpreting novelty detection in Section 4 . We conclude the paper in Section 5

\section{Generative kernel PCA}

In this section we give a short review on how kernel PCA can be used to generate new data, as introduced in [13. We start with the calculation of the kernel principal components for a $d$-dimensional dataset $\left\{x_{i}\right\}_{i=1}^{N}$ with $N$ data points and for each data point $x_{i} \in \mathbb{R}^{d}$. Compared to regular PCA, kernel PCA first maps the input data to a high dimensional feature space $\mathcal{F}$ using a feature map 
$\phi(\cdot)$. In this feature space, regular PCA is performed on the points $\phi\left(x_{i}\right)$ for $i=1, \ldots, N$. By using a kernel function $k(x, y)$ defined as the inner product $(\phi(x) \cdot \phi(y))$, an explicit expression for $\phi(\cdot)$ can be avoided. Typical examples of such kernels are given by the Gaussian RBF kernel $k(x, y)=e^{-\|x-y\|_{2}^{2} /\left(2 \sigma^{2}\right)}$ or the Laplace kernel $k(x, y)=e^{-\|x-y\|_{2} / \sigma}$, where $\sigma$ denotes the bandwidth. Finding the principal components amounts to solving the eigenvalue problem for the kernel matrix $K$, with matrix elements $K_{i j}=\left(\phi\left(\boldsymbol{x}_{i}\right) \cdot \phi\left(\boldsymbol{x}_{j}\right)\right)$. The eigenvalue problem for kernel PCA is stated as follows:

$$
K H^{\top}=H^{\top} \Lambda,
$$

where $H=\left[h_{1}, \ldots, h_{N}\right] \in \mathbb{R}^{d \times N}$, the first $d \leq N$ components are used, is the matrix with the eigenvectors in each column and $\Lambda=\operatorname{diag}\left\{\lambda_{1}, \ldots, \lambda_{d}\right\}$ the matrix with the corresponding eigenvalues on the diagonal. In the framework of RKMs, the points $\phi\left(x_{i}\right)$ correspond to visible units $v_{i}$ and $h_{i}$ are the hidden units. As in [13, we can now construct the generative equation:

$$
v^{\star}=\phi\left(x^{\star}\right)=\left(\sum_{i=1}^{N} \phi\left(x_{i}\right) h_{i}^{\top}\right) h^{\star}
$$

where $h^{\star}$ represents a newly generated hidden unit and $v^{\star}$ the corresponding visible unit. Finding $x^{\star}$ in Eq. (2) corresponds to the pre-image problem [6]. In [13, the authors give a possible solution by multiplying both sides with $\phi\left(x_{k}\right)$, which gives the output of the kernel function for the generated point in the input space $x^{\star}$ and the data point $x_{k}$ :

$$
\hat{k}\left(x_{k}, x^{\star}\right)=\sum_{i=1}^{N} k\left(x_{k}, x_{i}\right) h_{i}^{\top} h^{\star} .
$$

The above equation can be seen as the similarity between the newly generated point $x^{\star}$ and $x_{k}$. This expression can be used in a kernel smoother approach to find an estimate $\hat{x}$ for the generated data point $x^{\star}$ :

$$
\hat{x}=\frac{\sum_{i=1}^{S} \tilde{k}\left(x_{i}, x^{\star}\right) x_{i}}{\sum_{i=1}^{S} \tilde{k}\left(x_{i}, x^{\star}\right)},
$$

where $\tilde{k}\left(x_{i}, x^{\star}\right)$ is the scaled similarity between 0 and 1 calculated in (3) and $S$ the number of closest points based on the similarity $\tilde{k}\left(x_{i}, x^{\star}\right)$. Given a point in the latent space $h^{\star}$, we get an approximation for the corresponding point $\hat{x}$ in input space. This mechanism makes it possible to continuously explore the latent space.

\footnotetext{
${ }^{1}$ For simplicity, we assume that the mapped data are centered in $\mathcal{F}$. Otherwise, we have to go through the same algebra using $\tilde{\phi}(x):=\phi(x)-\sum_{i=1}^{N} \phi\left(x_{i}\right)$. This is the same assumption as in [12.
} 


\section{Latent space exploration}

Our goal is to use generative kernel PCA to explore the latent space. Therefore we developed a tool where generative kernel PCA can easily be utilised for new datasets. First kernel PCA is performed to find the hidden features of the dataset. After choosing an initial hidden unit as starting point, we vary the values for each component of the hidden unit to explore the latent space. The corresponding newly generated data point in the input space is estimated using the kernel smoother approach.

In the tool, a partial visualisation of the latent space projected onto two principal components is shown. We continuously vary the values of the components of the selected hidden unit. This allows the exploration of the extracted latent space by visualising the resulting variation in the input space. The ability to perform incremental variations aids interpretation of the meaning encoded in the latent space along a chosen direction. In Fig. 1, the interface of our tool is shown.
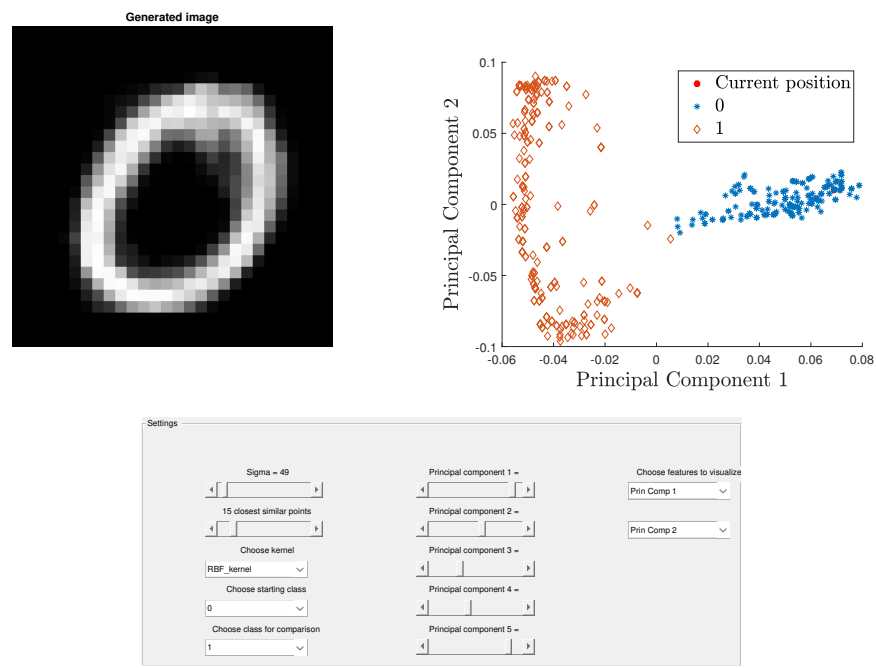

Fig. 1: Interface of the Matlab tool for generative kernel PCA. At the bottom, the parameter values and position in the latent space can be chosen. In the top right position, the latent space along two selected principal components is shown. In the top left, the newly generated data point in the input space is visualised for the MNIST data set.

As an example we use this tool to explore the latent space of the MNIST handwritten digits dataset [9], where we sample 1000 data points each of digits zero 
and one. We use a Gaussian kernel with bandwidth $\sigma^{2}=50, S=15$ and number of components $d=10$. In Fig. 2, the latent space is shown along the first two principal components as well as the first and third components.

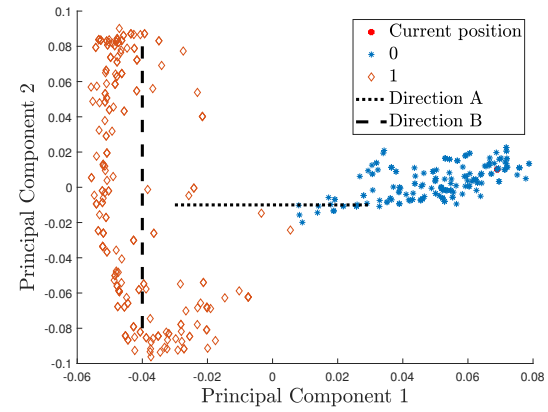

(a)

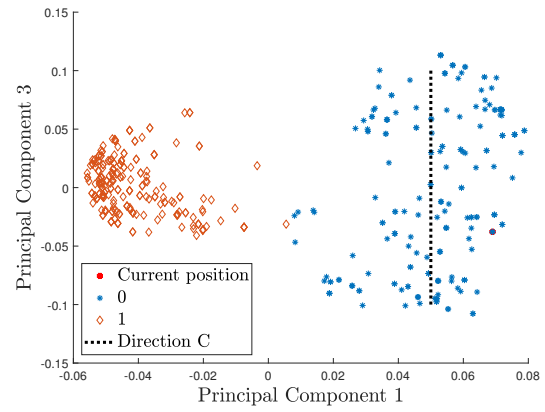

(b)

Fig. 2: Latent space of the MNIST digits dataset for the digits 0 and 1 . The dotted lines indicate the direction along which new data points are generated. (a) Data projected on the first two principal components (b) Data projected on the first and third principal component.
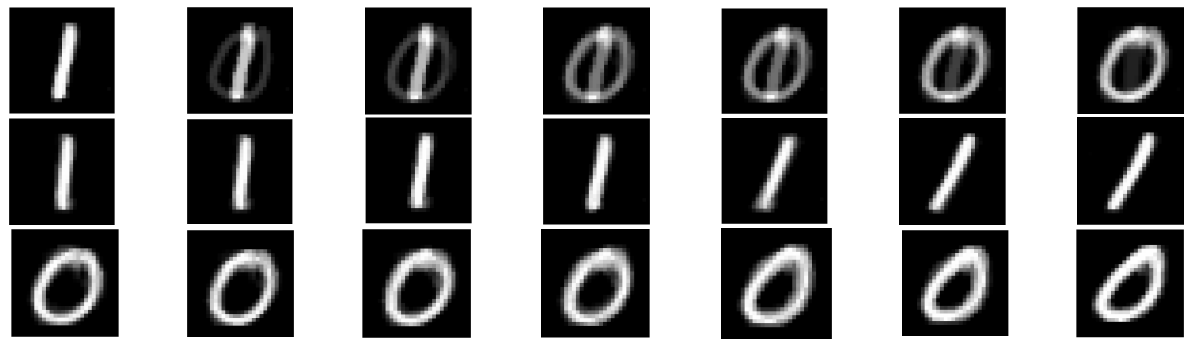

Fig. 3: Exploration of the latent space of the MNIST digits data set. In the top two rows images are generated along the directions A and B in Fig 2a and in the bottom row the images are generated along direction $\mathrm{C}$ in Fig. $2 \mathrm{~b}$

In Fig. 3, digits are generated along the directions indicated on the plots of the latent space in Fig. 2. This allows us to interpret the different regions and the meaning of the principal components. Along direction A, corresponding to the first principal component, we find an interpolation between the regions with digits of zero and one. Direction B seems to correlate with the orientation of the digit. This explains the smaller variation along the second principal component for the zeros as rotating the digit zero has a smaller effect compared to the 
rotation of digit one. The third direction, corresponding to component 3 , seems to be related to squeezing the zeros together, which explains the larger variance for the zeros compared to the ones.

Another example of latent space exploration is done on the Extended Yale Face Database B [1], where we sample 1720 data points. We use a Gaussian kernel with bandwidth $\sigma^{2}=650, S=45$ and number of components $d=20$. The latent space along the first two principal components is shown in Fig. 4e. Four different regions within the feature space are highlighted from which corresponding images are generated. The dissimilarity between the images in the various regions suggests the components capture different lighting conditions on the subjects. The tool allows us to gradually move between these different regions and see the changes in the input space as shown in Fig. 5. Moving between regions A and B shows a increasing illumination of the subject. We can thus interpret the first principal component as determining the global level of illumination. The bottom row seems to indicate that the second principal component can be interpreted as the position of the light source. In region $\mathrm{C}$ of the feature space the points are illuminated from the right and region $\mathrm{D}$ from the left.

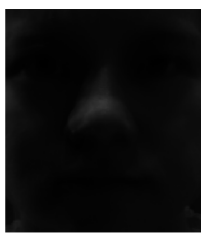

(a)

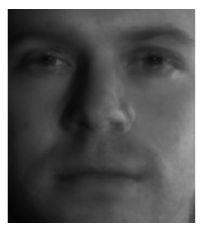

(c)

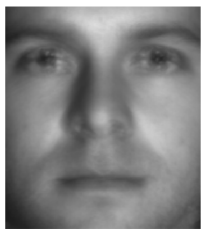

(b)

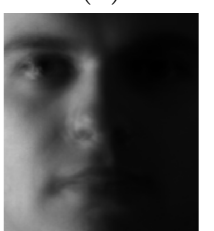

(d)

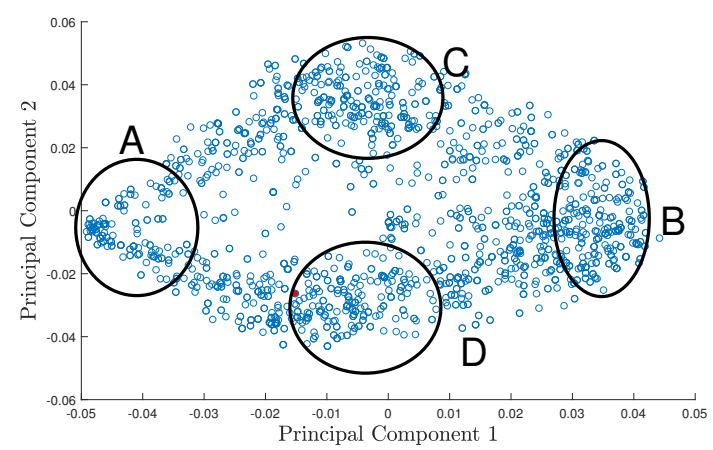

(e)

Fig. 4: Exploring different regions of the latent space of the Yale Face Database. (e) Data projected on the first two principal components for the Yale Face Database. (a)-(d) Generated faces from the different regions.

As a further example of generative kernel PCA, we use the tool to interpolate between 2 faces. Kernel PCA is performed on a subset of the database consisting out of 130 facial images of two subjects, the hyperparameters are the same as above. Variation along the fourth principal component results in a smooth interpolation between the two subjects, shown in Fig. 6 . 

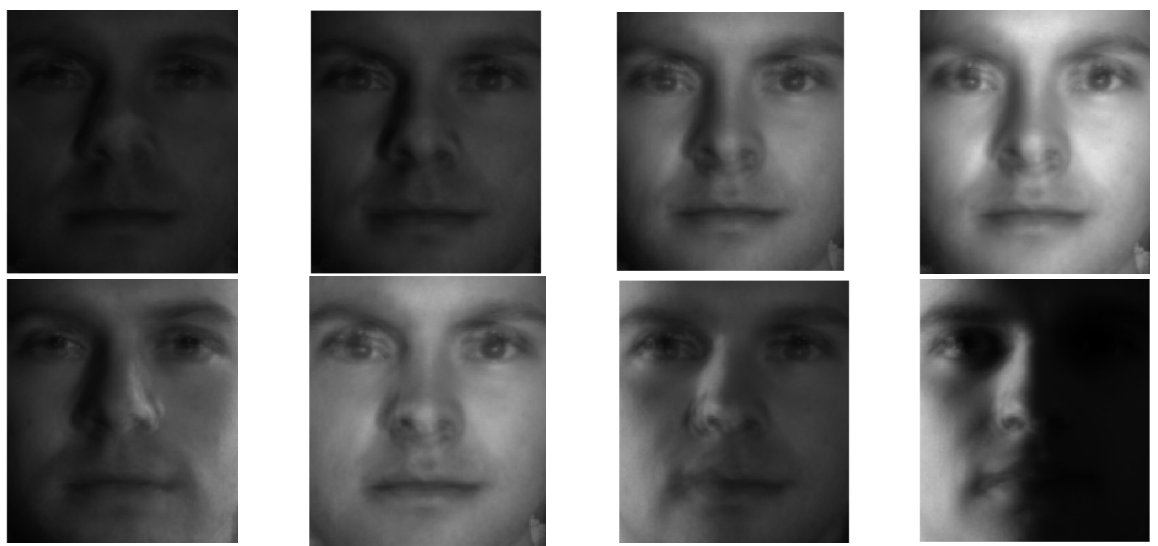

Fig. 5: Exploring the space between the regions of the latent space in figure 4e. The top row shows images generated between regions $\mathrm{A}$ and $\mathrm{B}$, while the bottom row explores the space between regions $\mathrm{C}$ and $\mathrm{D}$.
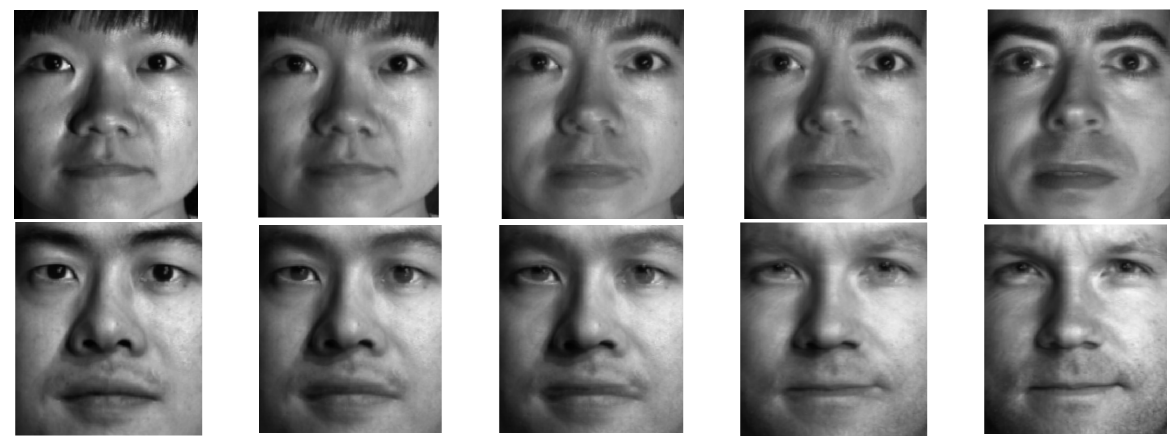

Fig. 6: Two examples of interpolation between two subjects of the Yale Face Database B along the fourth component.

\section{Novelty detection}

As a final illustration of latent space exploration using generative kernel PCA, we consider an application within the context of novelty detection. We use the reconstruction error in feature space as a measure of novelty [4, where Hoffmann shows the metric demonstrates a competitive performance on synthetic distributions and real-world data sets. The novelty score is calculated for all data points, where the $20 \%$ of data points with the largest novelty score are considered novel. These points typically reside in low density regions of the latent space and are highlighted as interesting regions to explore using the tool. we consider 1000 instances of the digit zero from the MNIST dataset. After performing kernel $\mathrm{PCA}$ with the same parameters as in the previous section, we explore the latent 
space around the detected novel patterns. The data projected on the first two principal components is shown in Fig. 7.

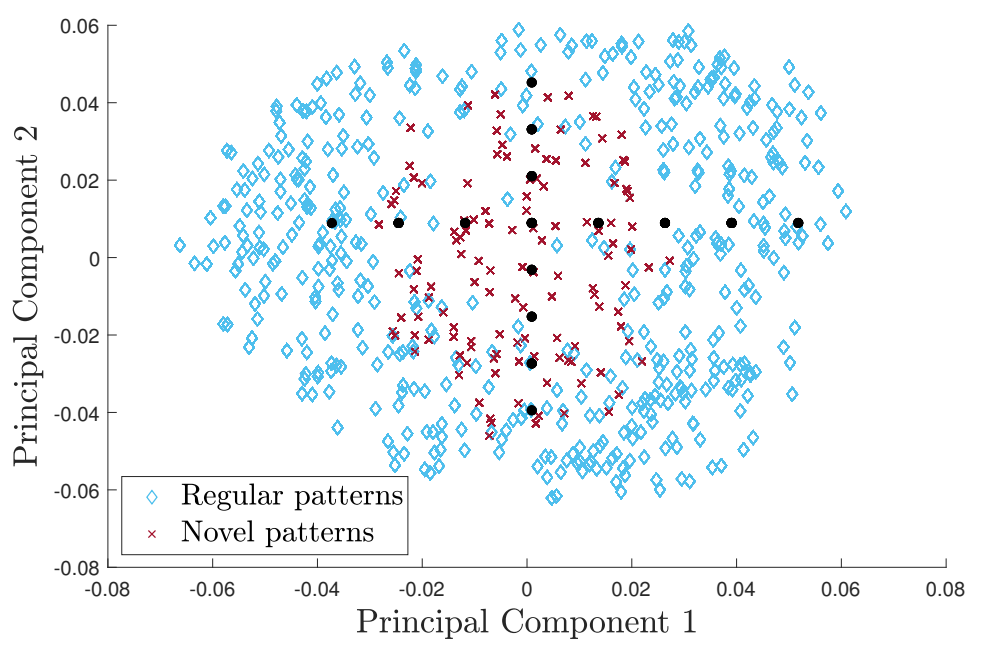

Fig. 7: The latent space for 1000 zeros of the MNIST digits data set. The central cluster of points consists out of data points with a high novelty score, this corresponds to a low density region in the latent space. The black dots indicate locations in the latent space from where new points are sampled for generative kernel PCA.

The generated images from the positions indicated by the black dots in Fig. 7 are shown in Fig. 8 The first row allows us to interpret the first principal component as moving from a thin round zero towards a more closed digit. The middle of the latent space is where the novel patterns are located which seems to indicate most zeros are either thin and wide or thick and narrow. A low amount of zeros in the data set are thick and wide or very thin and narrow. The bottom row of Fig. 8 gives the interpretation for the second principal component as rotating the digit. The novel patterns seem to be clustered more together and as such have a less obvious orientation. Important to note is that we only look at the first 2 components for the interpretation, while in practice the novelty detection method takes all 20 components into consideration.

Above experiment shows that latent space exploration methods can give additional insights for novelty detection. Both the generating mechanism, as well as the novelty detection make use of the kernel PCA formulation. The two methods naturally complement each other: the novelty detection provides interesting regions in the latent space to explore, at the same time helps the generative mechanism in interpreting why certain points are considered as novel. 

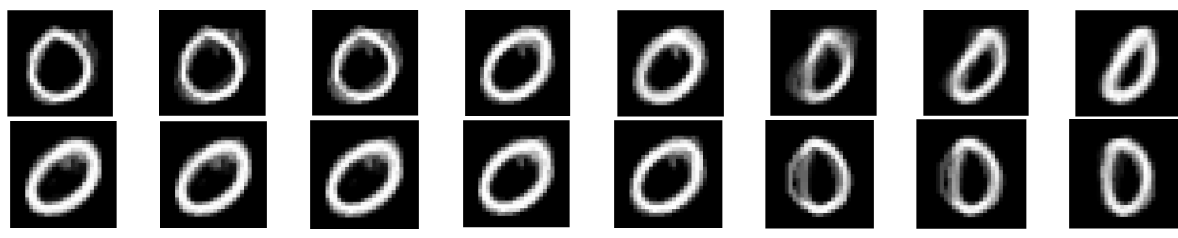

Fig. 8: Exploration of the latent space in Fig. 7. The top row indicates the points generated from the horizontal black dots, while the bottom row correspond to the vertical positions.

\section{Conclusion}

We showed the use of generative kernel PCA in exploring the latent space. Gradually moving along components in the feature space allows for the interpretation of components and consequently additional insight into the underlying latent space. This mechanism is demonstrated on the MNIST handwritten digits data set and the Yale Face Database B. As an illustration, feature space exploration is used in the context of novelty detection [5, where the latent space around novel patterns in data is explored. This to helps the interpretation of why certain points are considered as novel. Possible future directions would be the consideration of the geometry of the latent space. Not moving in straight lines, but curves trough high density regions. We would also like to explore more data sets and real-life examples using this newly developed tool.

\section{Acknowledgements}

EU: The research leading to these results has received funding from the European Research Council under the European Unions Horizon 2020 research and innovation program / ERC Advanced Grant E-DUALITY (787960). This paper reflects only the authors views and the Union is not liable for any use that may be made of the contained information. Research Council KUL: Optimization frameworks for deep kernel machines C14/18/068. Flemish Government: FWO: projects: GOA4917N (Deep Restricted Kernel Machines: Methods and Foundations), PhD/Postdoc grant. 


\section{References}

1. Georghiades, A., Belhumeur, P., Kriegman, D.: From few to many: Illumination cone models for face recognition under variable lighting and pose. IEEE Trans. Pattern Anal. Mach. Intelligence 23(6), 643-660 (2001)

2. Goodfellow, I., Pouget-Abadie, J., Mirza, M., Xu, B., Warde-Farley, D., Ozair, S., Courville, A., Bengio, Y.: Generative adversarial nets. In: Advances in neural information processing systems. pp. 2672-2680 (2014)

3. Hinton, G.E.: A practical guide to training restricted Boltzmann machines. In: Neural networks: Tricks of the trade, pp. 599-619. Springer (2012)

4. Hoffmann, H.: Kernel PCA for novelty detection. Pattern recognition 40(3), 863874 (2007)

5. Hofmann, T., Schölkopf, B., Smola, A.J.: Kernel methods in machine learning. The annals of statistics pp. 1171-1220 (2008)

6. Honeine, P., Richard, C.: Preimage problem in kernel-based machine learning. IEEE Signal Processing Magazine 28(2), 77-88 (2011)

7. Karras, T., Laine, S., Aila, T.: A style-based generator architecture for generative adversarial networks. In: Proceedings of the IEEE Conference on Computer Vision and Pattern Recognition. pp. 4401-4410 (2019)

8. Kingma, D.P., Welling, M.: Auto-encoding variational Bayes. arXiv preprint arXiv:1312.6114 (2013)

9. LeCun, Y., Bottou, L., Bengio, Y., Haffner, P., et al.: Gradient-based learning applied to document recognition. Proceedings of the IEEE 86(11), 2278-2324 (1998)

10. Liu, Y., Jun, E., Li, Q., Heer, J.: Latent space cartography: Visual analysis of vector space embeddings. In: Computer Graphics Forum. vol. 38, pp. 67-78. Wiley Online Library (2019)

11. Radford, A., Metz, L., Chintala, S.: Unsupervised representation learning with deep convolutional generative adversarial networks. arXiv preprint arXiv:1511.06434 (2015)

12. Schölkopf, B., Smola, A., Müller, K.R.: Kernel principal component analysis. In: International conference on artificial neural networks. pp. 583-588. Springer (1997)

13. Schreurs, J., Suykens, J.A.K.: Generative kernel pca. ESANN 2018 pp. 129-134 (2018)

14. Suykens, J.A.K.: Deep restricted kernel machines using conjugate feature duality. Neural computation 29(8), 2123-2163 (2017)

15. Way, G.P., Greene, C.S.: Extracting a biologically relevant latent space from cancer transcriptomes with variational autoencoders. BioRxiv p. 174474 (2017)

16. Wu, J., Zhang, C., Xue, T., Freeman, B., Tenenbaum, J.: Learning a probabilistic latent space of object shapes via $3 d$ generative-adversarial modeling. In: Advances in NeurIPS. pp. 82-90 (2016) 